\title{
Increased Body Mass Index Is a Risk Factor for Poor Clinical Outcomes after Radical Prostatectomy in Men with International Society of Urological Pathology Grade Group 1 Prostate Cancer Diagnosed with Systematic Biopsies
}

\author{
Matteo Ferro $^{a}$ Daniela Terracciano $^{\mathrm{b}}$ Gennaro Musia ${ }^{\mathrm{a}}$ Ottavio de Cobellia \\ Mihai Dorin Vartolomeic, d Rocco Damiano ${ }^{e}$ Francesco Cantiello $^{e}$ Carlo Buonerba $^{f}$ \\ Michele Morelli $^{a} \quad$ Francesco Alessandro Mistretta $^{a}$ Stefano Luzzago ${ }^{a}$ Sisto Perdonà ${ }^{g}$ \\ Paola Del Prete $^{\mathrm{h}}$ Francesco Del Giudice ${ }^{\mathrm{i}}$ Gian Maria Busetto ${ }^{\mathrm{i}}$ Angelo Porreca $^{\mathrm{j}}$ \\ Riccardo Autorinok $^{k}$ Matteo Manfredil Francesco Porpiglial Matteo Muto ${ }^{m}$ Davide Loizzo ${ }^{n}$ \\ Pasquale Ditonno $^{n}$ Michele Battaglian ${ }^{n}$ Giuseppe Lucarellin

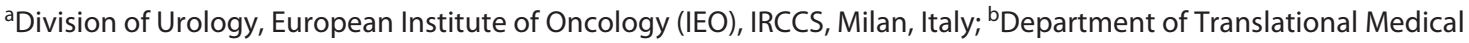 \\ Sciences, University of Naples "Federico II", Naples, Italy; 'Department of Urology, Comprehensive Cancer Center, \\ Vienna General Hospital, Medical University of Vienna, Vienna, Austria; ${ }^{\mathrm{d} D e p a r t m e n t}$ of Cell and Molecular Biology, \\ University of Medicine, Pharmacy, Sciences and Technology, Targu-Mures, Romania; 'Department of Urology, \\ Magna Graecia University of Catanzaro, Catanzaro, Italy; fDepartment of Oncology and Hematology, Regional \\ Reference Center for Rare Tumors, AOU Federico II of Naples, Naples, Italy; ${ }^{9}$ Division of Urology, Istituto Nazionale \\ Tumori di Napoli, IRCCS "G. Pascale", Naples, Italy; hScientific Directorate, Istituto Nazionale Tumori di Napoli, \\ IRCCS "G. Pascale", Naples, Italy; 'Department of Urology, Sapienza Rome University, Rome, Italy; 'Department \\ of Urology, Policlinico Abano Terme, Abano Terme, Italy; ${ }^{k}$ Division of Urology, VCU Health, Richmond, VA, USA; \\ 'Division of Urology, Department of Oncology, School of Medicine, San Luigi Hospital, University of Turin, Turin, \\ Italy; ${ }^{m}$ Department of Clinical Medicine and Surgery, Federico II University Medical School of Naples, Naples, Italy; \\ nepartment of Emergency and Organ Transplantation-Urology, Andrology and Kidney Transplantation Unit, \\ University of Bari, Bari, Italy
}

\section{Keywords}

Prostate cancer · Body mass index · Obesity · International

Society of Urological Pathology

\section{Abstract \\ Introduction: The association between obesity and clinically significant prostate cancer (PCa) is still a matter of debate. In this study, we evaluated the effect of body mass index (BMI) on the prediction of pathological unfavorable disease (UD), positive surgical margins (PSMs), and biochemical recur-}

rence $(B C R)$ in patients with clinically localized $(\leq C T 2 c)$ International Society of Urological Pathology (ISUP) grade group 1 PCa at biopsy. Methods: 427 patients with ISUP grade group 1 PCa who have undergone radical prostatectomy and BMI evaluation were included. The outcome of interest was the presence of UD (defined as ISUP grade group $\geq 3$ and PT $\geq 3 a$ ), PSM, and BCR. Results: Statistically significant differences resulted in comparing BMI with prostate-specific antigen (PSA) and serum testosterone levels (both $p<0.0001$ ). Patients with UD and PSM had higher BMI values $(p<0.0001$ and $p=0.006$, respectively). BCR-free survival was signifi- karger@karger.com

www.karger.com/uin Karger!
(C) 2021 S. Karger AG, Basel
Correspondence to:

Matteo Ferro, matteo.ferro@ieo.it

Giuseppe Lucarelli, giuseppe.lucarelli@inwind.it 
cantly decreased in patients with higher BMI values $(p<$ 0.0001 ). BMI was an independent risk factor for BCR and PSM. Receiver-operating characteristic analysis testing PSA accuracy in different BMI groups, showed that PSA had a reduced predictive value (area under the curve $[A \cup C]=0.535 ; 95 \%$ confidence interval $[\mathrm{Cl}]=0.422-0.646)$, in obese men compared to overweight (AUC $=0.664 ; 95 \% \mathrm{Cl}=0.598-0.725$ ) and normal weight patients (AUC $=0.721 ; 95 \% \mathrm{Cl}=0.660$ 0.777). Conclusion: Our findings show that increased $\mathrm{BMI}$ is a significant predictor of UD and PSM at RP in patients with preoperative low-to intermediate-risk diseases, suggesting that BMl evaluation may be useful in a clinical setting to identify patients with favorable preoperative disease characteristics harboring high-risk PCa.

c 2021 S. Karger AG, Basel

\section{Introduction}

More than 20 years ago, the World Health Organization identified obesity as a global health problem that has grown to epidemic proportions [1]. Many studies have demonstrated the negative impact of the excessive accumulation of body fat and various diseases and clinical conditions such as metabolic syndrome, type 2 diabetes, cardiovascular disease, obstructive sleep apnea, osteoarthritis, and liver disease [2-4]. Moreover, obesity has also been associated with increased risks of a number of cancers, including endometrial cancer, gastrointestinal cancers, meningioma, multiple myeloma, and urological tumors [5-9].

About prostate cancer (PCa), several mechanisms have been proposed to explain the association between obesity and its increased incidence and the risk of biochemical recurrence (BCR) after radical prostatectomy [10-12]. An opening question remains the understanding of mechanisms that regulate the prostate-specific antigen (PSA) levels and the biological aggressiveness of $\mathrm{PCa}$ in obese men. In addition, delayed diagnosis of PCa represents another critical issue in men with increased body mass index (BMI). The diagnostic delay may be caused by a variety of factors, including lower PSA values as an effect of hemodilution and lower levels of testosterone, as well as a consequence of a low diagnostic accuracy of prostate biopsies in larger prostate [13,14].

A delayed diagnosis of PCa in men with increased BMI might translate to a worse prognosis and might in part explain the high rate of capsular incision and positive surgical margin (PSM) at the time of radical prostatectomy, as well as an increased risk of BCR after surgery $[15,16]$.
In this study, we evaluated the effect of BMI on the prediction of pathological unfavorable disease (UD), PSMs, and BCR in patients with clinically localized $(\leq \mathrm{cT} 2 \mathrm{c})$ International Society of Urological Pathology (ISUP) grade group $1 \mathrm{PCa}$ at biopsy.

\section{Materials and Methods}

Patients

This study included 427 consecutive men with localized ISUP grade $1 \mathrm{PCa}$, who underwent laparoscopic or robot-assisted RP within 3 months from diagnosis, between January 2009 and December 2015. RP specimens were processed and evaluated according to the Stanford protocol [9] by 2-experienced genitourinary pathologists, blinded to the test results of each institution.

For all patients, biopsies were analyzed according to the 2014 ISUP recommendations $[17,18]$. Prostate biopsies were performed using a transrectal approach. During the procedure, an experienced urologist measured the prostate volume and carried out 12 18 core biopsies using an $18-\mathrm{G}$ Tru-Cut needle.

None of the study patients received neoadjuvant hormonal therapy (antiandrogens or luteinizing hormone-releasing hormone analogs or antagonists) or other hormonal preparations (i.e., 5 - $\alpha$ reductase inhibitors) that could alter their PSA values. We also excluded patients with acute bacterial prostatitis or previous prostate surgery in the 3 months before biopsy. In addition, subjects with chronic renal disease, alterations in blood protein levels at electrophoresis, hemophilia, incurable endocrine diseases, or those who had previously undergone multiple transfusions were excluded from the study because these conditions could alter the concentration of total PSA and testosterone. All patients underwent systematic blood sampling between 7:00 and 10:00 a.m. on the day before surgery to assess serum total testosterone concentrations.

Data collected included age, preoperative PSA level, PSA density, pathological stage, serum total testosterone, and BMI. For low-risk localized PCa, no additional imaging was recommended for staging purposes according to EAU guidelines [19].

The patients were stratified according to ISUP grade groups 1-5. BMI groups were defined by the WHO as follows: normal weight $\left(18.5-24.9 \mathrm{~kg} / \mathrm{m}^{2}, n=225\right)$, overweight $\left(25-29.9 \mathrm{~kg} / \mathrm{m}^{2}\right.$, $n=95)$, and obese $\left(>30 \mathrm{~kg} / \mathrm{m}^{2}, n=82\right)$. Disease upstaging was regarded as pathological stage $\geq \mathrm{T} 3 \mathrm{a}$ after RP with clinical stage $\leq \mathrm{T} 2 \mathrm{c}$. PCa upgrading was defined as ISUP grade group $\geq 3$ in RP specimens. UD was defined as the occurrence of pathological stage $\geq$ PT3 and ISUP grade group $\geq 3$ at RP specimens' pathology. BCR following RP was defined according to EAU guidelines.

The protocol for the research project has been approved by the local hospital Ethics Committees and conforms to the provisions of the 1995 Declaration of Helsinki. Written informed consent was obtained from all patients.

\section{Statistical Analysis}

Statistical calculations were performed with MedCalc 9.2.0.1 (MedCalc Software, Mariakerke, Belgium) and PASW 18 software (PASW 18; SPSS, Chicago, IL, USA). Comparisons of median values of the various variables between different groups were evaluated by Mann-Whitney $\mathrm{U}$ test or Kruskal-Wallis as appropriate. 
Table 1. Clinical and pathological characteristics by BMI

\begin{tabular}{|c|c|c|c|}
\hline Variable & $\begin{array}{l}\text { normal } \\
\text { BMI }<25 \mathrm{~kg} / \mathrm{m}^{2}\end{array}$ & $\begin{array}{l}\text { overweight } \\
\text { BMI } 25-29.9 \mathrm{~kg} / \mathrm{m}^{2}\end{array}$ & $\begin{array}{l}\text { obese } \\
\text { BMI }>30 \mathrm{~kg} / \mathrm{m}^{2}\end{array}$ \\
\hline Patients, $n$ & 225 & 95 & 82 \\
\hline Age at diagnosis, median (95\% CI) & $64(63-65)$ & $66(65-67.5)$ & $63(60-66)$ \\
\hline PSA, ng/mL at diagnosis, median (95\% CI) & $5.86(5.36-6.11)$ & $5.2(4.93-5.76)$ & $4.25(4.00-5.09)$ \\
\hline Testosterone, median (95\% CI) & $500(456-532)$ & $400(300-501)$ & $288(271-390)$ \\
\hline $\mathrm{Nr}$ of cores, median $(95 \% \mathrm{CI})$ & $12(12-12)$ & $14(14-14)$ & $14(12-14)$ \\
\hline $\mathrm{Nr}$ of positive cores, $2 ; n(\%)$ & $92(40.8)$ & $51(53.6)$ & $46(56.1)$ \\
\hline Max $\%$ of core involved by tumor, median $(95 \% \mathrm{CI})$ & $20(20-25)$ & $20(20-30)$ & $35(30-40)$ \\
\hline \multicolumn{4}{|l|}{ Pathological stage, $n(\%)$} \\
\hline 1 & $177(78.6)$ & $43(45.3)$ & $19(23.2)$ \\
\hline 2 & $24(10.7)$ & $24(25.3)$ & $18(21.9)$ \\
\hline 3 & $22(9.8)$ & $26(27.3)$ & $31(37.8)$ \\
\hline 4 & $2(0.9)$ & $2(2.1)$ & $4(4.9)$ \\
\hline 5 & 0 & 0 & $10(12.2)$ \\
\hline
\end{tabular}

BMI, body mass index; CI, confidence interval; ISUP, International Society of Urological Pathology.

The predictive accuracy of PSA and BMI for BCR was evaluated using receiver-operating characteristic (ROC) analysis and quantified in terms of area under the curve (AUC) and corresponding $95 \%$ confidence interval $(95 \% \mathrm{CI})$. Stepwise logistic regression was performed to determine factors influencing PSM.

Univariate and Multivariate analyses were performed using the Cox proportional hazards regression model to identify the most significant variables for predicting BCR. A backward selection procedure was performed with removal criterion $p>0.10$ based on likelihood ratio tests. Model calibration was measured by the Hosmer-Lemeshow goodness of fit test, with $p<0.05$ considered statistically significant.

Spearman test was applied to evaluate the correlations between BMI values, PSA, and testosterone. A $p$ value of $<0.05$ was considered statistically significant.

\section{Results}

Demographic and clinical characteristics of the overall study population are summarized in Table 1. PSA values and testosterone levels were lower in those patients classified as overweight or obese. An inverse correlation has been shown between PSA and BMI values (Spearman correlation: $\left.r_{\mathrm{s}}=-0.215, p<0.0001\right)$. Similarly, an inverse correlation has been observed between total testosterone levels and BMI $\left(r_{\mathrm{s}}=-0.244, p<0.0001\right)$. In addition, patients with UD and PSMs had higher BMI values $(p<$ 0.0001 and $p=0.006$, respectively) (Fig. 1a-f).

Kaplan-Meier survival curves for BCR-free survival, stratified by BMI groups, are shown in Figure 2. BCR-free survival was significantly decreased in patients with BMI $>30 \mathrm{~kg} / \mathrm{m}^{2}(p<0.0001)$. Logistic regression model showed that increased BMI and higher percentage of positive cores in biopsy specimens were risk factors for PSM (Table 2).

Multivariate analysis including age, BMI, PSA, PSM, and ISUP grade groups as covariates (Table 3 ) showed that BMI and PSA were significant independent predictors of BCR, with the Hosmer-Lemeshow statistics showing adequate model calibration $(p=0.24)$. ROC analysis testing the performance of PSA and BMI in predicting BCR showed that BMI had the highest AUC compared to PSA $(\mathrm{AUC}=0.792[95 \%$ confidence interval $(\mathrm{CI})=$ 0.750-0.829] vs. $\mathrm{AUC}=0.589$ [95\% CI $=0.541-0.636]$, respectively. $p<0.001)$. In addition, ROC analysis testing PSA accuracy in different BMI groups, showed that PSA had a reduced predictive value (AUC $=0.535 ; 95 \%$ CI $=$ 0.422-0.646) in obese men compared to overweight (AUC $=0.664 ; 95 \% \mathrm{CI}=0.598-0.725)$ and normal weight patients (AUC $=0.721 ; 95 \% \mathrm{CI}=0.660-0.777$ ). 


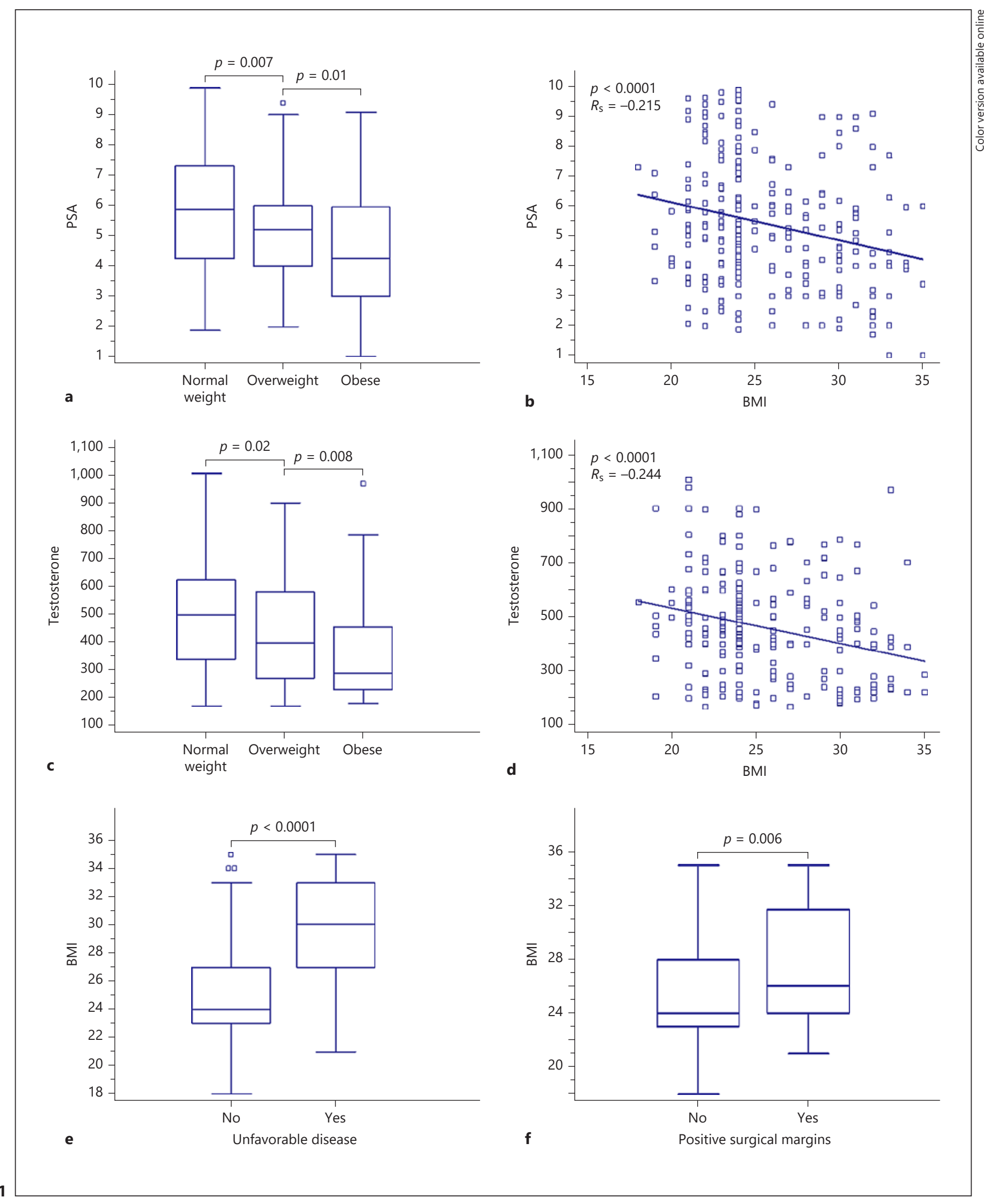

(For legend see next page.) 


\section{Discussion}

Altered adipose tissue homeostasis may be an important contributor to the development and/or progression of a number of solid organ tumors, including PCa [1012]. Recent evidence supports a positive association between obesity and increased risk of cancer, with stronger links to more aggressive disease [20-22]. Increased adiposity can sustain tumorigenesis by providing critical metabolites for cancer growth and pro-inflammatory cytokines, which are major drivers for many types of tumors, including PCa [23-25]. It has been shown that high BMI men have lower testosterone levels, which predispose them to more aggressive disease and poor prognosis $\mathrm{PCa}[13,14]$, and indeed, in our study, statistically significant differences resulted between BMI groups, total serum testosterone, PSM, and UD.

Furthermore, increasing evidences suggested that obesity, measured by BMI, is associated with increased risk of PCa-specific mortality and BCR [26-28]. In our cohort, BMI and PSA were significant independent predictors of BCR, and according to the Kaplan-Meier survival curves stratified by BMI groups, BCR-free survival was significantly decreased in patients with $\mathrm{BMI}>30 \mathrm{~kg} / \mathrm{m}^{2}$.

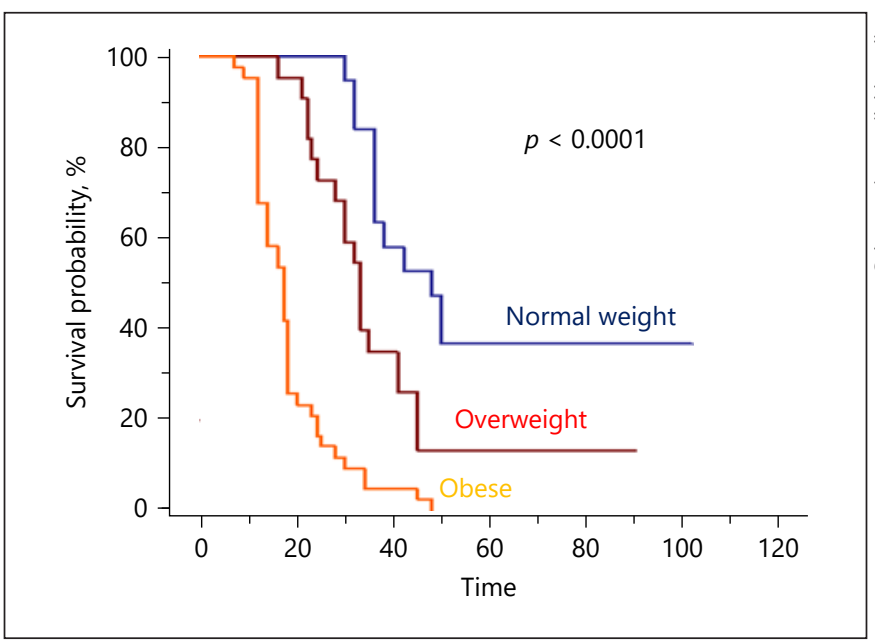

Fig. 2. Kaplan-Meier curves for BCR-free survival stratified by $\mathrm{BMI}$ groups. BMI, body mass index; $\mathrm{BCR}$, biochemical recurrence.
In addition, in our study, we found an increased rate of a pT3 disease $(29.3 \%)$ in obese men compared to normal or overweight patients (5-6\%). Several studies showed more adverse pathological features in obese men undergoing radical prostatectomy, and it is evident that obesity makes early detection of PCa more difficult due to less PSA screening, lower accuracy of digital rectal examination in obese men, and lower PSA values caused by obesity-related hemodilution $[29,30]$. As shown in the ROC analysis testing PSA accuracy in different BMI groups, PSA had a reduced predictive value in obese men compared to overweight and normal weight patients.

Nowadays, there is an attitude to extend active surveillance protocols not only to GG1 but also to GG2 disease with one high-grade core at the biopsy [31]; nevertheless, we have to be careful in the decision-making. In this scenario, recent studies showed that obesity is independently associated with a higher risk of pathological and therapeutic progression after initial biopsy [32], and data from another study support the idea that obesity is associated with PCa aggressiveness with a higher risk of upgraded and upstaged disease [33]. Therefore, active surveillance protocols in this subset of patients should include close surveillance scheme in order to early identify tumor progression.

Positive surgical margins play a key role in predicting clinical outcomes after RP. Yu et al. [34] demonstrated that obese patients showed a significant association with adverse surgical outcomes such as PSM, and according to the results of our survival analyses, it could be postulated that obesity-associated pathological variables simultaneously influenced BCR-free survival. Furthermore, obese patients had greater PSM and were statistically significant predictors of BCR-free survival after RP.

Current data would suggest that although BMI is a valid parameter, other criteria should be considered to stratify obese patients and their prognosis after RP. Adipose tissue and, in particular, the perivisceral fat, is metabolically active and has a particular inflammatory profile [35]. Recent studies have demonstrated that an increased thickness of the adipose tissue surrounding the neoplastic prostate (periprostatic adipose tissue [PPAT]) was associated with poor clinical outcomes and the presence of high-grade disease [36-39].
Fig. 1. a PSA levels stratified according to BMI groups. b Scatter diagram with regression line between BMI and PSA values. c Testosterone levels stratified according to BMI groups. d Scatter diagram with regression line between BMI and testosterone values. e Distribution of BMI according to the presence of UD. $\mathbf{f}$ Distribution of BMI according to the presence of PSMs. PSA, prostatespecific antigen; BMI, body mass index; UD, unfavorable disease; PSM, positive surgical margin. 
Table 2. Logistic regression model for PSMs

Table 3. Cox proportional hazards regression model for BCR prediction

\begin{tabular}{lccc}
\hline Variable & OR & $95 \%$ CI & $p$ value \\
\hline BMI & 1.95 & $1.15-3.28$ & 0.01 \\
$\begin{array}{l}\text { Percentage of positive cores in } \\
\quad \text { biopsy specimens }\end{array}$ & 1.81 & $1.16-3.02$ & 0.01 \\
PSA at diagnosis & 1.02 & $0.92-1.15$ & 0.68 \\
Prostate volume & 0.95 & $0.91-1.01$ & 0.21 \\
\hline
\end{tabular}

BMI, body mass index; CI, confidence interval; OR, odds ratio; PSA, prostate-specific antigen; PSM, positive surgical margin.

\begin{tabular}{|c|c|c|c|c|}
\hline \multirow[t]{2}{*}{ Variable } & \multicolumn{2}{|l|}{ Univariate } & \multicolumn{2}{|l|}{ Multivariate } \\
\hline & HR (95\% CI) & $p$ value & HR (95\% CI) & $p$ value \\
\hline Age & $1.04(1.01-1.12)$ & 0.01 & & - \\
\hline ISUP group & $2.53(1.72-3.96)$ & 0.01 & & - \\
\hline BMI & $1.18(1.09-1.42)$ & 0.0001 & $1.23(1.14-1.32)$ & 0.001 \\
\hline PSA & $1.09(1.02-1.12)$ & 0.0001 & $1.16(1.04-1.31)$ & 0.008 \\
\hline PSM & $2.21(2.04-3.28)$ & 0.01 & & - \\
\hline
\end{tabular}

BMI, body mass index; CI, confidence interval; PSA, prostate-specific antigen; PSM, positive surgical margins; ISUP, International Society of Urological Pathology; BCR, biochemical recurrence.
Salji et al. [40] first observed, by accurately quantifying the volume of PPAT by MRI, that PPAT volume measurement is a better prognostic tool for identifying patients at risk of developing castration-resistant PCa. The results from Sasaki et al. [41] showed that pretreatment ratio of PPAT to subcutaneous fat thickness on MRI is an independent survival predictor in hormone-naive men with advanced PCa. Interestingly, PPAT but not visceral fat was associated with OS in patients with advanced $\mathrm{PCa}$ at the time of diagnosis, opening to the future prospective that is moving along to evaluate patient's PPF thickness as a better prognostic tool [41].

The present study had some limitations. First, there might have been the inherent bias of a retrospective design and a selection bias. The data were analyzed in selected patients who underwent RP rather than all patients with biopsy-confirmed PCa. In addition, we used only BMI as a clinical parameter in order to evaluate overweight/obesity. In a recent article, Shachar et al. suggested to evaluate body composition beyond BMI and to use different parameters for a better risk stratification [42].

\section{Conclusions}

Our findings suggest that in patients with preoperative low-to intermediate-risk disease, an increased BMI is a predictor of unfavorable prognosis according to the most recently proposed PCa grading system. Therefore, BMI evaluation may be useful in a clinical setting to identify patients with favorable preoperative disease characteristics harboring high-risk PCa.

\section{Acknowledgments}

The authors wish to express their deepest gratitude to Fondazione Muto Onlus (Casavatore, Naples, Italy) to support the publication of this article.

\section{Statement of Ethics}

All procedures performed in studies involving human participants were in accordance with the ethical standards of the institutional and/or national research committee and with the $1964 \mathrm{Hel}-$ sinki Declaration and its later amendments or comparable ethical standards. The protocol for the research project has been approved by the local hospital Ethics Committees. Written informed consent was obtained from all individual participants included in the study. 


\section{Conflict of Interest Statement}

The authors have no conflicts of interest to declare.

\section{Funding Sources}

This research did not receive any specific grant from funding agencies in the public, commercial, or not-for-profit sectors.

\section{Author Contributions}

(1) Conception and design: F.M. and L.G.; (2) administrative support: F.M. and M.M.; (3) provision of study materials or patients: all the authors; (4) collection and assembly of data: F.M.; (5) data analysis and interpretation: L.G. and F.M.; (6) manuscript writing: all the authors; (7) final approval of manuscript: all the authors.

\section{References}

1 World Health Organization. Obesity: preventing and managing the global epidemic: report of a WHO consultation on obesity, Geneva, 3-5 June 1997. Geneva, Switzerland: World Health Organization; 1998.

2 Jensen MD. Role of body fat distribution and the metabolic complications of obesity. J Clin Endocrinol Metab. 2008;93(11 Suppl 1):S5763

3 Despres JP, Lemieux I. Abdominal obesity and metabolic syndrome. Nature. 2006;444: 881-7.

4 Mathieu P, Poirier P, Pibarot P, Lemieux I, Després JP. Visceral obesity: the link among inflammation, hypertension, and cardiovascular disease. Hypertension. 2009;53:577-84

5 Calle EE, Kaaks R. Overweight, obesity and cancer: epidemiological evidence and proposed mechanisms. Nat Rev Cancer. 2004;4: 579-91.

6 Ferro M, Vartolomei MD, Russo GI, Cantiello F, Farhan ARA, Terracciano D, et al. An increased body mass index is associated with a worse prognosis in patients administered BCG immunotherapy for T1 bladder cancer. World J Urol. 2019;37(3):507-14.

7 Ferro M, Katalin MO, Buonerba C, Marian R Cantiello F, Musi G, et al. Type 2 diabetes mellitus predicts worse outcomes in patients with high-grade $\mathrm{T} 1$ bladder cancer receiving bacillus Calmette-Guérin after transurethral resection of the bladder tumor. Urol Oncol. 2020;38(5):459-64.

8 Ferro M, Di Lorenzo G, Buonerba C, Lucarelli G, Russo GI, Cantiello F, et al. Predictors of residual $\mathrm{T} 1$ high grade on re-transurethral resection in a large multi-institutional cohort of patients with primary T1 high-grade/grade 3 bladder cancer. J Cancer. 2018;9(22):4250-4. Published 2018 Oct 20.

9 Lucarelli G, Loizzo D, Franzin R, Battaglia S, Ferro M, Cantiello F, et al. Metabolomic insights into pathophysiological mechanisms and biomarker discovery in clear cell renal cell carcinoma. Expert Rev Mol Diagn. 2019; 19(5):397-407.

10 Lucarelli G, Rutigliano M, Galleggiante V, Giglio A, Palazzo S, Ferro M, et al. Metabolomic profiling for the identification of novel diagnostic markers in prostate cancer. Expert Rev Mol Diagn. 2015;15(9):1211-24.
11 Ferro M, Terracciano D, Buonerba C, Lucarelli $\mathrm{G}$, Bottero D, Perdonà $\mathrm{S}$, et al. The emerging role of obesity, diet and lipid metabolism in prostate cancer. Future Oncol. 2017; 13(3):285-93.

12 Lucarelli G, Loizzo D, Ferro M, Rutigliano M, Vartolomei MD, Cantiello F, et al. Metabolomic profiling for the identification of novel diagnostic markers and therapeutic targets in prostate cancer: an update. Expert Rev Mol Diagn. 2019;19(5):377-87.

13 Ferro M, Lucarelli G, Bruzzese D, Di Lorenzo G, Perdonà $S$, Autorino R, et al. Low serum total testosterone level as a predictor of upstaging and upgrading in low-risk prostate cancer patients meeting the inclusion criteria for active surveillance. Oncotarget. 2017; 8(11):18424-34.

14 Ferro M, Lucarelli G, de Cobelli O, Vartolomei MD, Damiano R, Cantiello F, et al. Circulating preoperative testosterone level predicts unfavourable disease at radical prostatectomy in men with International Society of Urological Pathology Grade Group 1 prostate cancer diagnosed with systematic biopsies. World J Urol. 2020. Published online ahead of print 2020 Jul 18.

15 Rundle A, Wang Y, Sadasivan S, Chitale DA, Gupta NS, Tang D, et al. Larger men have larger prostates: detection bias in epidemiologic studies of obesity and prostate cancer risk. Prostate. 2017;77(9):949-54.

16 Freedland SJ, Grubb KA, Yiu SK, Nielsen ME, Mangold LA, Isaacs WB, et al. Obesity and capsular incision at the time of open retropubic radical prostatectomy. J Urol. 2005;174(5): 1798-801

17 Epstein JI, Egevad L, Amin MB, Delahunt B, Srigley JR, Humphrey PA. The 2014 international society of urological pathology (ISUP) consensus conference on Gleason grading of prostatic carcinoma: definition of grading patterns and proposal for a new grading system. Am J Surg Pathol. 2016;40(2):244-52.

18 Epstein JI, Amin MB, Reuter VE, Humphrey PA. Contemporary Gleason grading of prostatic carcinoma: an update with discussion on practical issues to implement the 2014 international society of urological pathology (ISUP) consensus conference on Gleason grading of prostatic carcinoma. Am J Surg Pathol. 2017;41(4):e1-7.
19 Heidenreich A, Bellmunt J, Bolla M, Joniau S, Mason M, Matveev V, et al. EAU guidelines on prostate cancer. Part 1: screening, diagnosis, and treatment of clinically localised disease. Eur Urol. 2011;59(1):61-71.

20 Calle EE, Rodriguez C, Walker-Thurmond K, Thun MJ. Overweight, obesity, and mortality from cancer in a prospectively studied cohort of U.S. adults. N Engl J Med. 2003;348(17): 1625-38.

21 Finley DS, Calvert VS, Inokuchi J, Lau A, Narula N, Petricoin EF, et al. Periprostatic adipose tissue as a modulator of prostate cancer aggressiveness. J Urol. 2009;182(4):1621-7.

22 von Hafe P, Pina F, Pérez A, Tavares M, Barros $\mathrm{H}$. Visceral fat accumulation as a risk factor for prostate cancer. Obes Res. 2004 Dec; 12(12):1930-5.

23 Lucarelli G, Ferro M, Loizzo D, Bianchi C Terracciano D, Cantiello F, et al. Integration of lipidomics and transcriptomics reveals reprogramming of the lipid metabolism and composition in clear cell renal cell carcinoma. Metabolites. 2020;10(12):509. Published 2020 Dec 13.

24 Netti GS, Lucarelli G, Spadaccino F, Castellano G, Gigante M, Divella C, et al. PTX3 modulates the immunoflogosis in tumor microenvironment and is a prognostic factor for patients with clear cell renal cell carcinoma. Aging. 2020;12(8):7585-602.

25 Huang J, Duran A, Reina-Campos M, Valencia T, Castilla EA, Müller TD, et al. Adipocyte p62/SQSTM1 suppresses tumorigenesis through opposite regulations of metabolism in adipose tissue and tumor. Cancer Cell. 2018;33(4):770-84.e6.

26 MacInnis RJ, English DR. Body size and composition and prostate cancer risk: systematic review and meta-regression analysis. Cancer Causes Control. 2006;17(8):989-1003.

27 Cao Y, Ma J. Body mass index, prostate cancer-specific mortality, and biochemical recurrence: a systematic review and meta-analysis. Cancer Prev Res. 2011;4(4):486-501.

28 Rodriguez C, Patel AV, Calle EE, Jacobs EJ, Chao A, Thun MJ. Body mass index, height, and prostate cancer mortality in two large cohorts of adult men in the United States. Cancer Epidemiol Biomarkers Prev. 2001;10(4): $345-53$. 
29 Freedland SJ, Sun L, Kane CJ, Presti JC, Terris MK, Amling CL, et al. Obesity and oncological outcome after radical prostatectomy: impact of prostate-specific antigen-based prostate cancer screening: results from the Shared Equal Access Regional Cancer Hospital and Duke Prostate Center databases. BJU Int. 2008;102(8):969-74.

30 Bañez LL, Hamilton RJ, Partin AW, Vollmer RT, Sun L, Rodriguez C, et al. Obesity-related plasma hemodilution and PSA concentration among men with prostate cancer. JAMA. 2007;298(19):2275-80.

31 Balakrishnan AS, Cowan JE, Cooperberg MR, Shinohara K, Nguyen HG, Carroll PR. Evaluating the safety of active surveillance: outcomes of deferred radical prostatectomy after an initial period of surveillance. J Urol. 2019; 202(3):506-10.

32 Bhindi B, Kulkarni GS, Finelli A, Alibhai SM, Hamilton RJ, Toi A, et al. Obesity is associated with risk of progression for low-risk prostate cancers managed expectantly. Eur Urol. 2014;66(5):841-8.
33 de Cobelli O, Terracciano D, Tagliabue E, Raimondi S, Galasso G, Cioffi A, et al. Body mass index was associated with upstaging and upgrading in patients with low-risk prostate cancer who met the inclusion criteria for active surveillance. Urol Oncol. 2015;33(5): 201-8.

$34 \mathrm{Yu}$ YD, Byun SS, Lee SE, Hong SK. Impact of body mass index on oncological outcomes of prostate cancer patients after radical prostatectomy. Sci Rep. 2018;8(1):11962. Published 2018 Aug 10.

35 Park J, Euhus DM, Scherer PE. Paracrine and endocrine effects of adipose tissue on cancer development and progression. Endocr Rev. 2011;32(4):550-70.

36 Bassett JK, Severi G, Baglietto L, MacInnis RJ, Hoang HN, Hopper JL, et al. Weight change and prostate cancer incidence and mortality. Int J Cancer. 2012;131(7):1711-9.

37 Bhindi B, Trottier G, Elharram M, Fernandes KA, Lockwood G, Toi A, et al. Measurement of peri-prostatic fat thickness using transrectal ultrasonography (TRUS): a new risk factor for prostate cancer. BJU Int. 2012;110(7): 980-6.
38 van Roermund JG, Hinnen KA, Tolman CJ, Bol GH, Witjes JA, Bosch JL, et al. Periprostatic fat correlates with tumour aggressiveness in prostate cancer patients. BJU Int. 2011; 107(11):1775-9.

39 Woo S, Cho JY, Kim SY, Kim SH. Periprostatic fat thickness on MRI: correlation with Gleason score in prostate cancer. AJR Am J Roentgenol. 2015;204(1):W43-7.

40 Salji M, Hendry J, Patel A, Ahmad I, Nixon C, Leung HY. Peri-prostatic fat volume measurement as a predictive tool for castration resistance in advanced prostate cancer. Eur Urol Focus. 2018;4(6):858-66.

41 Sasaki T, Sugino Y, Kato M, Nishikawa K, Kanda H. Pre-treatment ratio of periprostatic to subcutaneous fat thickness on MRI is an independent survival predictor in hormonenaive men with advanced prostate cancer. Int J Clin Oncol. 2020;25(2):370-6.

42 StrulovShachar S, Williams GR. The obesity paradox in cancer-moving beyond BMI. Cancer Epidemiol Biomarkers Prev. 2017;26(1): 13-6. 